OPEN ACCESS

Edited by:

Francesco Cosentino, University of Molise, Italy

Reviewed by: Diana Butera, University Hospital of Parma, Italy Carmine Conte, Agostino Gemelli University Polyclinic (IRCCS), Italy

*Correspondence: Xiaoxie Wang fwf20110322@163.com

Specialty section: This article was submitted to Obstetrics and Gynecological Surgery,

a section of the journal

Frontiers in Medicine

Received: 25 December 2021 Accepted: 02 February 2022 Published: 01 March 2022

Citation:

Yao G, Qiu J, Zhu F and Wang X

(2022) Survival of Patients With

Cervical Cancer Treated With Definitive Radiotherapy or Concurrent Chemoradiotherapy According to Histological Subtype: A Systematic

Review and Meta-Analysis.

Front. Med. 9:843262.

doi: 10.3389/fmed.2022.843262

\section{Survival of Patients With Cervical Cancer Treated With Definitive Radiotherapy or Concurrent Chemoradiotherapy According to Histological Subtype: A Systematic Review and Meta-Analysis}

\author{
Guorong Yao, Jian Qiu, Fengjia Zhu and Xiaoxie Wang* \\ Department of Gynaecology and Obstetrics, Huzhou Central Hospital, Affiliated Central Hospital Huzhou University, Huzhou, \\ China
}

Background: Cervical cancer is a leading cause of morbidity and mortality for women worldwide. Different histopathological cervical cancer subtypes (i.e., adenocarcinoma/adenosquamous carcinoma, and squamous cell carcinoma) are all treated similarly with definitive radiotherapy or concurrent chemoradiotherapy, but studies have reported differing survival prognoses. In this review and meta-analysis, we compared the disease-free and overall survivals of patients with cervical cancer treated with definitive radiotherapy or concurrent chemoradiotherapy according to the histopathological subtypes.

Objective: To compare the disease-free and overall survivals of patients with adenocarcinoma/adenosquamous carcinoma and squamous cell carcinoma cervical cancer treated with definitive radiotherapy or concurrent chemoradiotherapy.

Methods: We systematically searched the Web of Science, EMBASE, CENTRAL, Scopus, and MEDLINE academic databases following PRISMA guidelines. We identified publications to conduct a random-effects meta-analysis to evaluate the disease-free and overall survivals of patients with cervical adenocarcinoma/adenosquamous carcinoma and squamous cell carcinoma treated with definitive radiotherapy or concurrent chemoradiotherapy.

Results: From 963 studies, we found eight eligible ones with 13,859 patients with cervical cancer (mean age, $52.2 \pm 7.9$ years). Our meta-analysis revealed a poorer outcome of disease-free (hazard ratio, 1.51; 95\% Cl, 1.28-1.79) and overall (hazard ratio $1.41 ; 95 \% \mathrm{Cl}, 1.26-1.57)$ survivals for patients with adenocarcinoma/adenosquamous carcinoma undergoing definitive radiotherapy or concurrent chemoradiotherapy than for those with squamous cell carcinoma undergoing similar treatments. We also observed that larger tumor size and advanced tumor stage are also significant prognostic factors that adversely impact survival outcomes in cervical cancer patients undergoing definitive radiotherapy or concurrent chemoradiotherapy. 
Conclusion: Our results show poor disease-free and overall survivals for patients with cervical cancer and adenocarcinoma/adenosquamous carcinoma than for those with squamous cell carcinoma after treatment with definitive radiotherapy or concurrent chemoradiotherapy. Our findings clarify the risks associated with the conventional management of cervical cancer according to the histological type.

Keywords: cervical cancer survival cervical cancer, chemotherapy, radiotherapy, disease-free survival, overall survival

\section{INTRODUCTION}

Cervical cancer is the fourth most common type of cancer in women $(1,2)$. According to the American Cancer Society, the malignancy originates in the cellular lining of the cervix, and most cases can be classified as being squamous cell carcinomas, with adenocarcinoma/adenosquamous carcinomas following the list $(3,4)$. Epidemiological studies have reported a high incidence of cervical cancer (almost 40\%) (5, 6), and the World Health Organization acknowledges that almost 310,000 women worldwide perish annually due to cervical cancer (2).

Past decades have largely seen a reduction in the incidence of the commonly occurring squamous cell carcinoma-based cervical cancer (7). This decrease in incidence has been attributed to the development of advanced cytological screening procedures that allow clinicians to preemptively treat the malignancy in its precancerous stages $(8-10)$. Despite the efficacy of such widespread screening programs, they are ineffective in detecting the other histopathological cervical cancer variants, and the overall incidence of cervical adenocarcinoma/adenosquamous carcinomas has increased worldwide (11-13). Randomized controlled trials have led to the use of concurrent chemotherapy alongside radiotherapy as the standard treatment for all the cervical cancer histopathological subtypes (14-16). However, this may not be the best approach $(17,18)$. However, some evidence suggests that this standard treatment may not be the best for patients with adenocarcinoma/adenosquamous carcinoma-based cervical cancer $(19,20)$.

Many cohort studies have compared the disease-free and overall survivals in patients with adenocarcinoma/adenosquamous carcinoma and squamous cell carcinoma $(17,18,20-24)$, their results differ as to the impact of the histopathological subtypes on the overall survival in these patients after definitive radiotherapy or concurrent chemoradiotherapy. Some studies found worse survivals for patients with adenocarcinoma/adenosquamous carcinoma than for those with squamous cell carcinoma $(18,20,21)$. But others found the worse survivals in the patients with squamous cell carcinoma (17, 22-24). Similarly, whether the disease-free survival differs among the patients with cervical cancer based on the histological type also remains unclear. Some studies found a stronger negative impact on the disease-free survival of patients with adenocarcinoma/adenosquamous carcinoma than on those with squamous cell carcinoma $(18,20,21)$, but others reported a lack of statistically significant differences $(17,23,24)$.
To the best of our knowledge, no systematic review or meta-analysis before this one has compared the survivals after definitive radiotherapy or concurrent chemoradiotherapy in patients with cervical cancer and either adenocarcinoma/adenosquamous carcinoma or squamous cell carcinoma. Therefore, we will attempt to synthesize the evidence on the disease-free and overall survivals of patients with cervical cancer according to their histopathological subtypes. Our findings should clarify the survival prognoses of the different cervical cancer subtypes after definitive radiotherapy or concurrent chemoradiotherapy.

\section{METHODS}

We adhered to the PRISMA (Preferred Reporting Items for Systematic Reviews and Meta-Analyses) guidelines (25) to conduct this meta-analysis.

\section{Data Search Strategy}

We searched the literature in five scientific databases (Web of Science, MEDLINE, CENTRAL, EMBASE, and Scopus) from inception until April 2021. The search was performed using a combination of $\mathrm{MeSH}$ keywords including "adenocarcinoma," "adenosquamous carcinoma," "squamous cell carcinoma," "cervical cancer," "radiation therapy," "concurrent chemoradiotherapy" included "disease-free survival," and "overall survival." We also manually searched the bibliography section of the studies to identify all relevant studies. The inclusion criteria follow:

a) Studies comparing the disease-free and/or overall survivals in between cervical cancer patients with adenocarcinoma/adenosquamous carcinoma and squamous cell carcinoma.

b) Studies with cervical cancer patients receiving radiation therapy and concurrent chemoradiotherapy.

c) Studies conducted in human participants.

d) Studies published in peer-reviewed scientific journals.

e) Studies published in English language.

Case series, case reports, conference proceedings and abstracts, letters to the editor, opinion papers, theses, reviews, and metaanalyses were not considered for this review. The screening of the studies was independently performed by two reviewers. Disagreements were resolved by discussion with a third independent reviewer. 


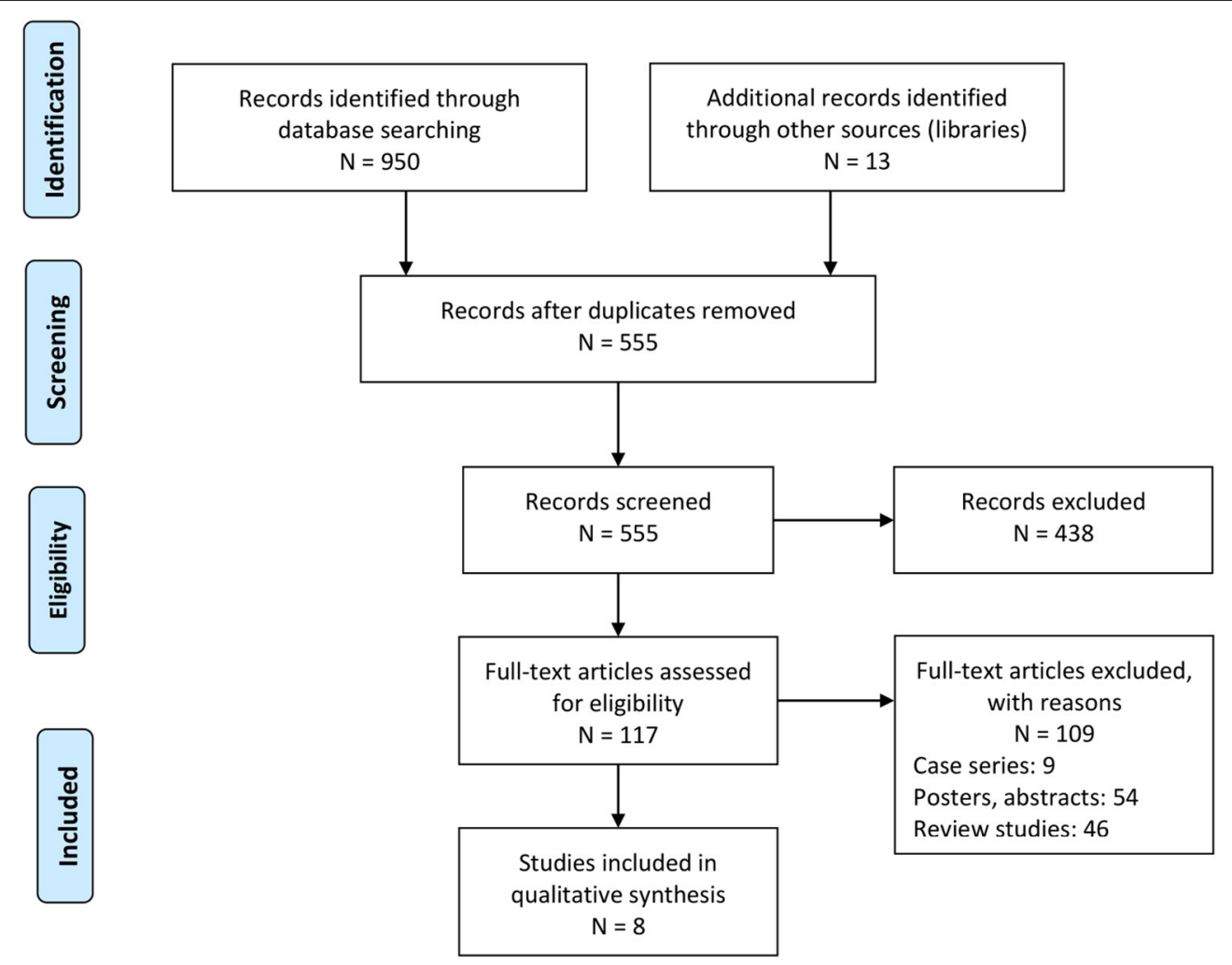

FIGURE 1 | PRISMA flowchart.

\section{Quality Assessment}

We assessed the risk of bias in the included studies using the Newcastle Ottawa scale (26). This tool evaluates the outcomes for selective reporting, confounding bias, measurement of outcomes, and incomplete data availability as bias threats. In addition, two reviewers independently appraised the methodological quality of the studies. Here, again disagreements were resolved after the intervention of a third reviewer to arbitrate.

\section{Data Analysis}

We used the Comprehensive Meta-analysis version 2.0 (CMA) software (27) to perform a within-group random-effects model meta-analysis (28). We calculated the hazard ratios to assess disease-free and overall survivals of patients with adenocarcinoma/adenosquamous carcinoma or squamous cell carcinoma. We assessed the heterogeneity among the studies by computing $I^{2}$ statistics; we considered values between 0 and $25 \%$ as indicative of negligible heterogeneity, values between 25 and $75 \%$ as moderately heterogeneous, and values $\geq 75 \%$ as substantially heterogeneous (29). We converted medians and ranges in individual studies into means and standard deviations using the method of Hozo et al. (30). Moreover, we applied Duval and Tweedy's trim and fill procedure (31) to evaluate publication bias. This publication bias analysis is characterized by the imputation of studies from either side of a plotted graph to identify any unbiased effects. The significance level for this study was determined at $5 \%$.

\section{RESULTS}

Our literature search provided a total of 950 studies. We identified an additional 13 during the screening of the reference sections of the included studies (Figure 1). After applying our inclusion criteria, we obtained eight retrospective cohort studies (17, 18, 20-24, 32). We extracted the data into tables (see summary in Table 1).

\section{Participant Information}

We included data from 13,859 women in the eight studies included. We found 11,891 patients with squamous cell carcinoma and 1,968 with adenocarcinoma/adenosquamous carcinoma.

The average age of the participants was as $52.2 \pm 7.9$ years. The average age of patients with squamous cell carcinoma was $55.01 \pm 6.58$ years, and the average age of patients with adenocarcinoma/adenosquamous carcinoma was $49.8 \pm 8.6$ years. Two studies failed to report the ages of their patients $(18,24)$.

\section{Quality Assessment for Cohort Studies}

Table 2 shows the results of the risk of bias obtained with the Newcastle Ottawa scale. We found the overall risk to be low (see also the graph on Figure 2). 
TABLE 1 | Details of the studies included in the meta-analysis.

\begin{tabular}{|c|c|c|c|c|c|c|c|c|c|c|}
\hline Study & Country & $\begin{array}{l}\text { Type of } \\
\text { study }\end{array}$ & $\begin{array}{l}\text { Sample } \\
\text { descriptive }\end{array}$ & $\begin{array}{l}\text { Age } \\
(\mathrm{M} \pm \mathrm{SD} \\
\text { years) }\end{array}$ & $\begin{array}{l}\text { Tumor } \\
\text { stage }\end{array}$ & $\begin{array}{l}\text { Tumor size } \\
\text { (cm) }\end{array}$ & $\begin{array}{l}\text { Duration of } \\
\text { radiation } \\
\text { therapy } \\
\text { (days) }\end{array}$ & $\begin{array}{l}\text { Follow-up } \\
\text { (months) }\end{array}$ & $\begin{array}{l}\text { Disease-free } \\
\text { survival } \\
\%(95 \% \mathrm{Cl})\end{array}$ & $\begin{array}{l}\text { Overall survival } \\
\%(95 \% \mathrm{Cl})\end{array}$ \\
\hline $\begin{array}{l}\text { Kang et al. } \\
\text { (21) }\end{array}$ & South Korea & $\begin{array}{l}\text { Retrospective } \\
\text { cohort study }\end{array}$ & $\begin{array}{l}\text { SCC: } 354 \\
\text { AC: } 44\end{array}$ & $\begin{array}{l}\text { SCC: } 57.4 \pm \\
12.0 \\
\text { AC: } 56.5 \\
\pm 12.2 \\
\end{array}$ & $\begin{array}{l}\text { SCC } \\
\|: 206 \\
I I I: 110 \\
\text { IV:38 } \\
\text { AC } \\
\|: 26 \\
I I I: 13 \\
I V: 5\end{array}$ & $\begin{array}{l}\text { SCC } \\
\geq 4: 84 \\
<4: 270 \\
\text { AC } \\
\geq 4: 13 \\
<4: 31\end{array}$ & $\begin{array}{l}\text { SCC: } 54 \\
\text { AC: } 53\end{array}$ & 60 & - & - \\
\hline Hu et al. (18) & China & $\begin{array}{l}\text { Retrospective } \\
\text { cohort study }\end{array}$ & $\begin{array}{l}\text { SCC: } 744 \\
\text { AC: } 71\end{array}$ & - & $\begin{array}{l}\text { SCC } \\
\text { I:92 } \\
\text { II:505 } \\
\text { III-IV:147 } \\
\text { AC } \\
\text { I:7 } \\
\text { II:54 } \\
\text { III-IV:10 }\end{array}$ & $\begin{array}{l}\text { SCC } \\
\geq 4: 457 \\
<4: 287 \\
\text { AC } \\
\geq 4: 42 \\
<4: 29\end{array}$ & - & 36.2 & $\begin{array}{l}\text { SCC: } 77.5 \% \\
\text { AC: } 57.3 \%\end{array}$ & $\begin{array}{l}\text { SCC: } 85.2 \% \\
\text { AC: } 75.4 \%\end{array}$ \\
\hline $\begin{array}{l}\text { Zhou et al. } \\
\text { (24) }\end{array}$ & China & $\begin{array}{l}\text { Retrospective } \\
\text { cohort study }\end{array}$ & $\begin{array}{l}\text { SCC: } 7,530 \\
\text { AC: } 925 \\
\text { ASC: } 296\end{array}$ & $52(19-98)$ & $\begin{array}{l}\text { SCC } \\
\text { I:1,589 } \\
\text { II:3,487 } \\
\text { III:2,235 } \\
\text { IV:219 } \\
\text { AC } \\
\text { I:296 } \\
\text { II:414 } \\
\text { III:190 } \\
\text { IV:25 } \\
\text { ASC } \\
\text { I: } 79 \\
\text { II:141 } \\
\text { III:64 } \\
\text { IV:12 }\end{array}$ & - & - & 39 & $\begin{array}{l}\text { SCC: } 59.3 \% \\
\text { AC/ASC: } 53.9 \%\end{array}$ & $\begin{array}{l}\text { SCC: } 51.1 \% \\
\text { AC/ASC: } 40.3 \%\end{array}$ \\
\hline $\begin{array}{l}\text { Rose et al. } \\
\text { (23) }\end{array}$ & Canada & $\begin{array}{l}\text { Retrospective } \\
\text { cohort study }\end{array}$ & $\begin{array}{l}\text { SCC: } 1,489 \\
\text { AC/ASC: } 182\end{array}$ & $\begin{array}{l}\text { SCC: } 46.5 \\
\text { AC/ASC: } 46\end{array}$ & $\begin{array}{l}\text { SCC } \\
\|: 1,000 \\
\text { III:446 } \\
\text { IV:43 } \\
\text { AC/ACS } \\
\text { II:136 } \\
\text { III:42 } \\
\text { IV:4 }\end{array}$ & $\begin{array}{l}\text { SCC } \\
\geq 5: 1,243 \\
<5: 234 \\
\text { AC/ACS } \\
\geq 5: 41 \\
<5: 141\end{array}$ & - & 120 & $\begin{array}{l}\text { SCC: } 52 \% \\
\text { AC/ASC: } 53.2 \%\end{array}$ & $\begin{array}{l}\text { SCC: } 48.2 \% \\
\text { AC/ASC: } 50.5 \%\end{array}$ \\
\hline $\begin{array}{l}\text { Chen et al. } \\
\text { (17) }\end{array}$ & Taiwan & $\begin{array}{l}\text { Retrospective } \\
\text { cohort study }\end{array}$ & $\begin{array}{l}\text { SCC: } 194 \\
\text { AC/ASC: } 35\end{array}$ & $\begin{array}{l}\text { SCC: } 63 \\
\text { AC/ASC: } 35\end{array}$ & $\begin{array}{l}\text { SCC } \\
\text { II:134 } \\
\text { III:50 } \\
\text { IV:10 } \\
\text { AC/ACS } \\
\text { II:26 } \\
\text { III:6 } \\
\text { IV:3 }\end{array}$ & $\begin{array}{l}\text { SCC } \\
\geq 5: 92 \\
<5: 102 \\
\text { AC/ACS } \\
\geq 5: 11 \\
<5: 24\end{array}$ & - & 60 & $\begin{array}{l}\text { SCC: } 47.6 \% \\
\text { AC/ASC: } 30 \%\end{array}$ & $\begin{array}{l}\text { SCC: } 58.1 \% \\
\text { AC/ASC: } 41.3 \%\end{array}$ \\
\hline
\end{tabular}


TABLE 1 | Continued

\begin{tabular}{|c|c|c|c|c|c|c|c|c|c|c|}
\hline Study & Country & $\begin{array}{l}\text { Type of } \\
\text { study }\end{array}$ & $\begin{array}{l}\text { Sample } \\
\text { descriptive }\end{array}$ & $\begin{array}{l}\text { Age } \\
(\mathrm{M} \pm \mathrm{SD} \\
\text { years) }\end{array}$ & $\begin{array}{l}\text { Tumor } \\
\text { stage }\end{array}$ & $\begin{array}{l}\text { Tumor size } \\
\text { (cm) }\end{array}$ & $\begin{array}{l}\text { Duration of } \\
\text { radiation } \\
\text { therapy } \\
\text { (days) }\end{array}$ & $\begin{array}{c}\text { Follow-up } \\
\text { (months) }\end{array}$ & $\begin{array}{l}\text { Disease-free } \\
\text { survival } \\
\%(95 \% \mathrm{Cl})\end{array}$ & $\begin{array}{l}\text { Overall survival } \\
\%(95 \% \mathrm{Cl})\end{array}$ \\
\hline Noh et al. (32) & South Korea & $\begin{array}{l}\text { Retrospective } \\
\text { cohort study }\end{array}$ & $\begin{array}{l}\text { SCC: } 1,073 \\
\text { AC: } 185 \\
\text { ASC: } 65\end{array}$ & $\begin{array}{l}\text { SCC: } 51 \\
\text { AC: } 46 \\
\text { ASC: } 50\end{array}$ & $\begin{array}{l}\text { SCC } \\
\text { I:757 } \\
\text { II:316 } \\
\text { AC } \\
\text { I:148 } \\
\|: 37 \\
\text { ASC } \\
\text { I:58 } \\
\|: 7\end{array}$ & - & - & 60 & - & $\begin{array}{l}\text { SCC: } 87.6 \% \\
\text { AC: } 75.8 \% \\
\text { ASC: } 83.2 \%\end{array}$ \\
\hline $\begin{array}{l}\text { Katanyoo } \\
\text { et al. (22) }\end{array}$ & Thailand & $\begin{array}{l}\text { Retrospective } \\
\text { cohort study }\end{array}$ & $\begin{array}{l}\text { SCC: } 282 \\
\text { ASC: } 141\end{array}$ & $\begin{array}{l}\text { SCC: } 50.8 \pm \\
10.7 \\
\text { ASC: } 49.1 \\
\pm 10.3\end{array}$ & $\begin{array}{l}\text { SCC } \\
\text { II:170 } \\
\text { III:110 } \\
\text { IV:2 } \\
\text { AC } \\
\text { II:85 } \\
\text { II: } 55 \\
\text { IV:1 }\end{array}$ & $\begin{array}{l}\text { SCC } \\
>4: 130 \\
\leq 4: 152 \\
\text { AC } \\
>4: 68 \\
\leq 4: 73\end{array}$ & - & 60 & - & $\begin{array}{l}\text { SCC: } 59.9 \% \\
\text { ASC: } 61.1 \%\end{array}$ \\
\hline
\end{tabular}

M, mean; SD, standard deviation; F, woman; M, man; SCC, squamous cell carcinoma; AC/ASC, adenocarcinoma/adenosquamous carcinoma.

TABLE 2 | Risk of bias for individual studies based on the Newcastle Ottawa scale.

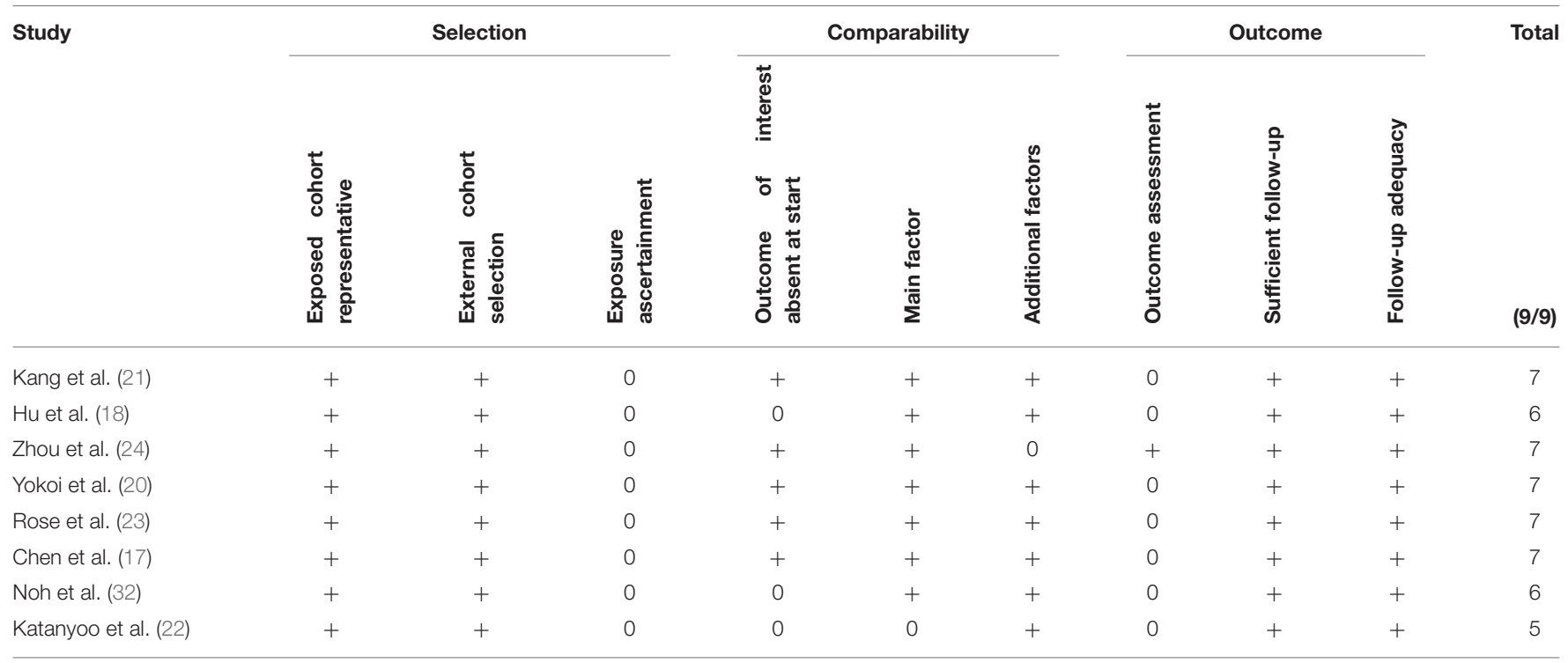

\section{Publication Bias}

We used Duval and Tweedy's trim and fill method to determine whether studies were missing on either side of the mean effect of the funnel plot due to publication bias. We identified three studies missing on the left side of the mean effect. The overall random effect models determined the point estimate (1.41) and the 95\% CI (1.26-1.57) for all the combined studies; after using the trim and fill method, the imputed point estimate was 1.32 and the 95\% CI (1.18-1.48). Figure 3 shows the publication bias graph.

\section{Meta-Analysis Report}

\section{Disease-Free Survival}

Six studies in our analysis reported disease-free survival statistics. We observed an increased hazard ratio in patients with adenocarcinoma/adenosquamous carcinoma than in patients with squamous cell carcinoma (Figure 4; hazard ratio, 1.51; $95 \% \mathrm{CI}, 1.28-1.79 ; p=0.001$ ) with moderate heterogeneity $\left(I^{2}: 44.2 \%\right)$.

We also conducted further subgroup analysis in which we evaluated disease-free survival outcome based on tumor size and the stage of tumor. Four studies had reported disease-free 


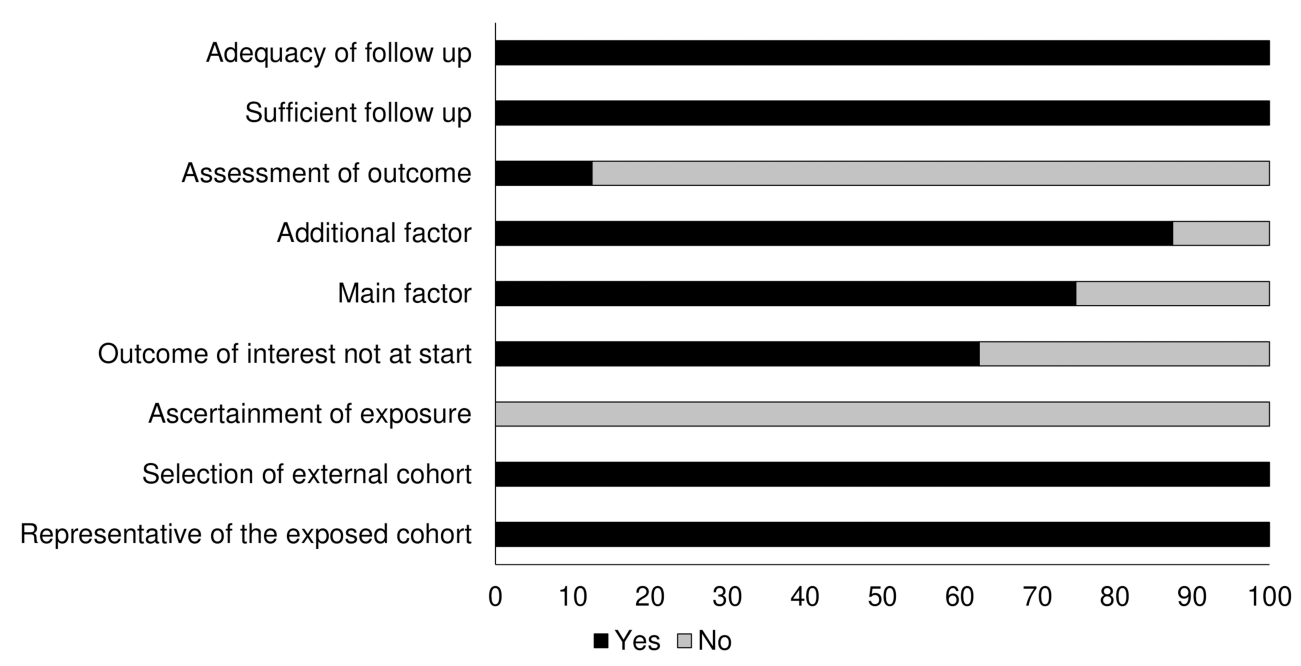

FIGURE 2 | Risk of bias according to the Newcastle Ottawa scale for cohort studies.

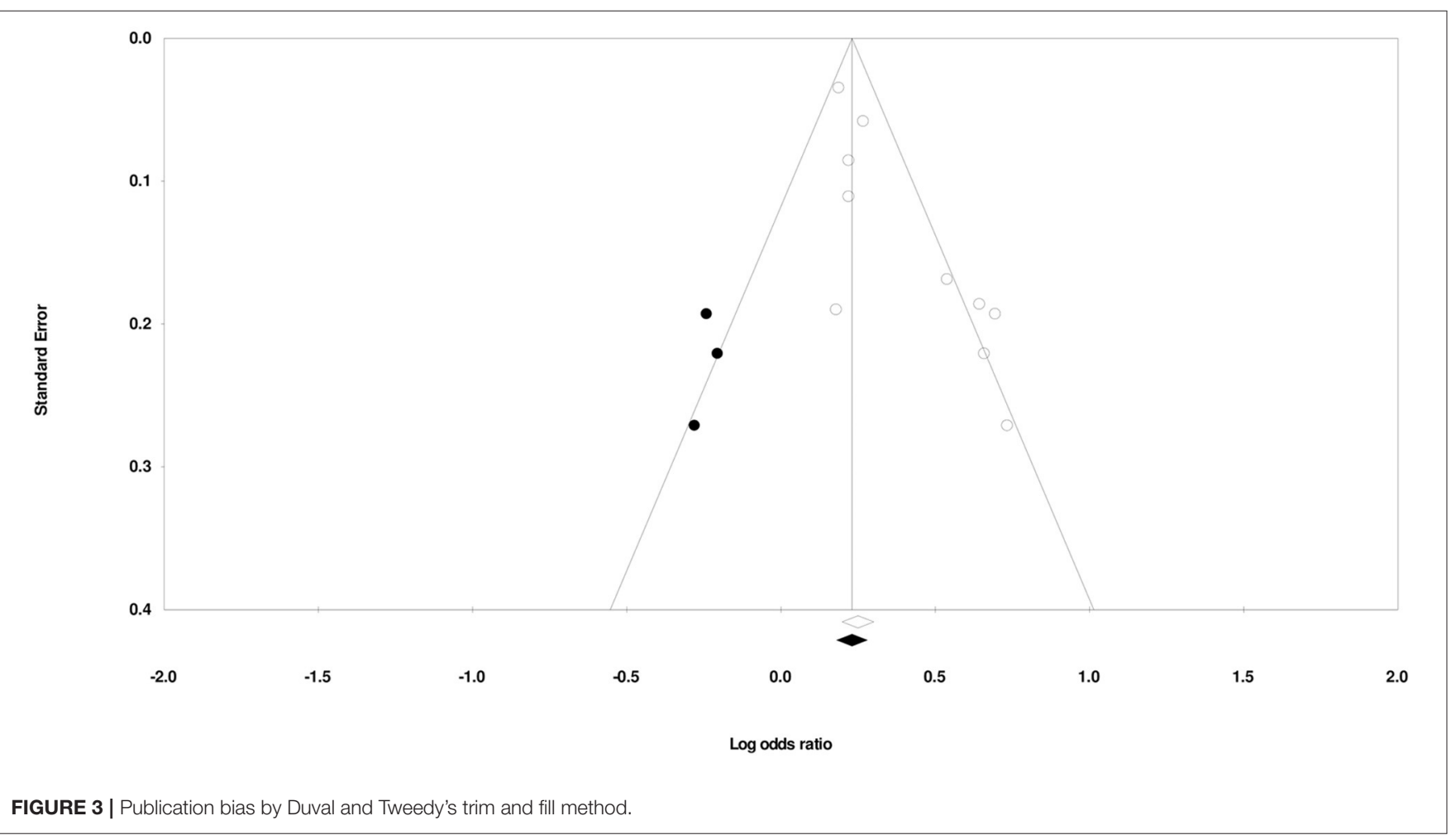

survival outcome based on the tumor size. We observed an increase hazard ratio in patients with larger tumor size than in patients with smaller (Figure 5; hazard ratio, 1.46; 95\% CI, $1.26-1.70 ; p=0.001)$ with no heterogeneity $\left(I^{2}: 0 \%\right)$. Five studies had reported disease-free survival outcome based on the tumor stage. We observed an increase hazard ratio in patients with higher tumor stage than in patients with lesser stage (Figure 6; hazard ratio, 1.90; 95\% CI, 0.92-3.95; $p=0.08$ ) with no heterogeneity $\left(I^{2}: 0 \%\right)$.

\section{Overall Survival}

The overall survival was reported by eight studies. We observed an increased hazard ratio in patients with adenocarcinoma/adenosquamous carcinoma than in patients with squamous cell carcinoma (Figure 7) (hazard ratio, 1.41; 95\% CI, 1.26-1.57; $p<0.001)$ with moderate heterogeneity $\left(I^{2}: 24.9 \%\right)$.

We conducted further subgroup analysis in which we evaluated overall survival outcome based on tumor size and 


\section{Study name}

Kang et al. (2020) AC

Hu et al. (2018) AC

Zhou et al. (2017) AC

Zhou et al. (2017) ASC

Yokoi et al. (2017) AC/ASC

Rose et al. (2014) AC/ASC

Chen et al. (2014) AC/ASC
Statistics for each study

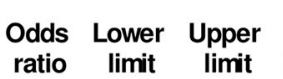

$\begin{array}{lll}2.370 & 1.628 & 3.450\end{array}$

$\begin{array}{lll}2.210 & 1.643 & 2.973\end{array}$

$\begin{array}{lll}1.280 & 1.178 & 1.391\end{array}$

$\begin{array}{lll}1.350 & 1.180 & 1.545\end{array}$

$\begin{array}{lll}2.200 & 1.480 & 3.271\end{array}$

$\begin{array}{lll}1.210 & 1.026 & 1.426\end{array}$

$\begin{array}{lll}1.130 & 0.801 & 1.595\end{array}$

$\begin{array}{lll}1.519 & 1.284 & 1.796\end{array}$
Z-Value p-Value

$\begin{array}{ll}4.505 & 0.000 \\ 5.238 & 0.000 \\ 5.836 & 0.000 \\ 4.367 & 0.000 \\ 3.897 & 0.000 \\ 2.272 & 0.023 \\ 0.696 & 0.487 \\ 4.885 & 0.000\end{array}$

\section{Odds ratio and $95 \% \mathrm{Cl}$}

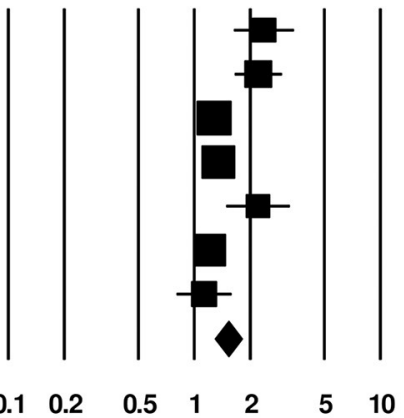

FIGURE 4 | Forest plot for studies evaluating the disease-free survival in patients with adenocarcinoma/adenosquamous carcinoma or squamous cell carcinoma.

Study name

$\begin{array}{lccccc} & \begin{array}{c}\text { Hazard } \\ \text { ratio }\end{array} & \begin{array}{c}\text { Lower } \\ \text { limit }\end{array} & \begin{array}{c}\text { Upper } \\ \text { limit }\end{array} & \text { Z-Value } & \text { p-Value } \\ \text { Kang et al. (2020) } & 1.640 & 1.133 & 2.374 & 2.622 & 0.009 \\ \text { Hu et al. (2018) } & 1.800 & 1.383 & 2.343 & 4.369 & 0.000 \\ \text { Rose et al. (2014) } & 1.320 & 1.127 & 1.546 & 3.448 & 0.001 \\ \text { Noh et al. (2014) } & 1.350 & 1.056 & 1.726 & 2.393 & 0.017 \\ & 1.465 & 1.260 & 1.703 & 4.959 & 0.000\end{array}$

Hazard ratio and $95 \% \mathrm{Cl}$

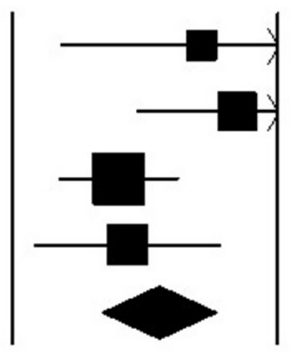

1

FIGURE 5 | Forest plot for studies evaluating the disease-free survival in patients with differential tumor size.

the stage of tumor. Four studies had reported overall survival outcome based on the tumor size. We observed an increase hazard ratio in patients with larger tumor size than in patients with smaller (Figure 8; hazard ratio, 1. 38; 95\% CI, 1.05-1.82; $p=$ $0.02)$ with no heterogeneity $\left(I^{2}: 0 \%\right)$. Eight studies had reported overall survival outcome based on the tumor stage. We observed an increase hazard ratio in patients with higher tumor stage than in patients with lesser stage (Figure 9; hazard ratio, 1.84; 95\% CI, $1.16-2.91 ; p=0.009)$ with no heterogeneity $\left(I^{2}: 0 \%\right)$.

\section{DISCUSSION}

We present evidence pointing to a difference in the survival of patients with cervical cancer undergoing definitive radiotherapy or concurrent chemoradiotherapy based on their histological type. We found poorer disease-free and overall survivals for patients with adenocarcinoma/adenosquamous carcinoma than for those with squamous cell carcinoma after definitive radiotherapy or concurrent chemoradiotherapy. We also observed that larger tumor size and advanced tumor stage are also significant prognostic factors that adversely impacts survival outcomes in in cervical cancer patients undergoing definitive radiotherapy or concurrent chemoradiotherapy.

Cervical cancer management is challenging because of its poor prognosis and various manifestations (33-35). Patients with cervical cancer are often treated with a similar approach (i.e., concurrent chemo- and radiotherapy) (14-16) regardless of the cancer's histopathological subtype. Some studies have suggested that patients with adenocarcinoma/adenosquamous cervical carcinoma do not fare as well as those with the squamous cell carcinoma subtype when treated with the standard therapy $(18,22)$. Moreover, some evidence suggests that the patients with adenocarcinoma/adenosquamous cervical carcinoma treated with the standard therapy may exhibit higher morbidity (high failure and relapse rates, 
Study name

$\begin{array}{lcccrr} & \begin{array}{c}\text { Hazard } \\ \text { ratio }\end{array} & \begin{array}{c}\text { Lower } \\ \text { limit }\end{array} & \begin{array}{c}\text { Upper } \\ \text { limit }\end{array} & \text { Z-Value } & \text { p-Value } \\ \text { Kang et al. (2020) } & 1.470 & 0.917 & 2.356 & 1.601 & 0.109 \\ \text { Hu et al. (2018) } & 1.560 & 1.275 & 1.909 & 4.317 & 0.000 \\ \text { Zhou et al. (2017) } & 3.880 & 3.384 & 4.448 & 19.449 & 0.000 \\ \text { Rose et al. (2014) } & 3.460 & 2.496 & 4.797 & 7.447 & 0.000 \\ \text { Chen et al. (2014) } & 0.820 & 0.714 & 0.942 & -2.797 & 0.005 \\ & 1.908 & 0.920 & 3.955 & 1.737 & 0.082\end{array}$

Hazard ratio and $95 \% \mathrm{Cl}$

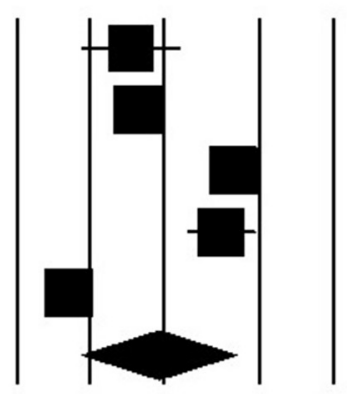

\section{$\begin{array}{lllllll}0.1 & 0.2 & 0.5 & 1 & 2 & 5 & 10\end{array}$}

FIGURE 6 | Forest plot for studies evaluating the disease-free survival in patients with differential tumor stage.

\section{Study name}

Kang et al. (2020) AC/ASC

Hu et al. (2018) AC/ASC

Zhou et al. (2017) AC

Zhou et al. (2017) ASC

Yokoi et al. (2017) AC/ASC

Rose et al. (2014) AC/ASC

Chen et al. (2014) AC/ASC

Noh et al. (2014) AC

Noh et al. (2014) ASC

Katanyoo et al. (2012) AC/ASC

\section{Statistics for each study}

Odds Lower Upper

ratio limit limit Z-Value $p$-Value

$\begin{array}{lllll}1.910 & 1.324 & 2.755 & 3.463 & 0.001\end{array}$

$\begin{array}{lllll}2.010 & 1.375 & 2.938 & 3.603 & 0.000\end{array}$

$\begin{array}{lllll}1.210 & 1.129 & 1.297 & 5.386 & 0.000\end{array}$

$\begin{array}{lllll}1.310 & 1.167 & 1.470 & 4.590 & 0.000\end{array}$

$\begin{array}{lllll}1.940 & 1.257 & 2.995 & 2.991 & 0.003\end{array}$

$\begin{array}{lllll}1.250 & 1.055 & 1.480 & 2.585 & 0.010\end{array}$

$\begin{array}{lllll}1.200 & 0.826 & 1.744 & 0.956 & 0.339\end{array}$

$\begin{array}{lllll}1.720 & 1.234 & 2.398 & 3.200 & 0.001\end{array}$

$\begin{array}{lllll}2.090 & 1.227 & 3.561 & 2.711 & 0.007\end{array}$

$\begin{array}{lllll}1.250 & 1.005 & 1.555 & 2.000 & 0.045\end{array}$

$\begin{array}{lllll}1.411 & 1.262 & 1.577 & 6.054 & 0.000\end{array}$

\section{Odds ratio and $95 \% \mathrm{Cl}$}

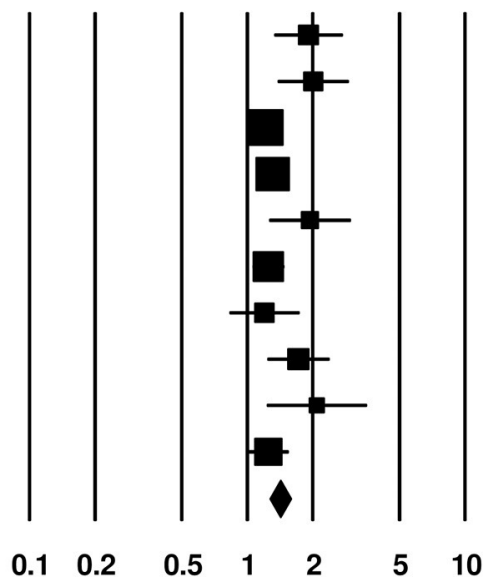

FIGURE 7 | Forest plot for studies evaluating the overall survival in patients with adenocarcinoma/adenosquamous carcinoma or squamous cell carcinoma.

lymph node metastases) and mortality rates (18) than their counterparts with squamous cell cervical carcinoma after the same treatments.

We observed that all the studies included had reported poorer disease-free survivals for the patients with adenocarcinoma/adenosquamous carcinoma than for those with squamous cell carcinoma-based cervical cancer. In a cohort representative of the Chinese population, Zhou et al. (24) found poorer disease-free survival in patients with adenocarcinoma/adenosquamous cervical carcinoma than in those with squamous cell cervical carcinoma. The authors attributed their findings and those by Huang et al. (36) to the radioresistant properties of adenocarcinoma/adenosquamous carcinomas, which prevent the cancer's complete cure, and ultimately affect the survival of the patients. Similarly, $\mathrm{Hu}$ et al. (18) also found a poorer 3-year disease-free survival rate for the adenocarcinoma group (53.7\%) than that for the squamous cell carcinoma group (77.5\%), and they confirmed their findings with a propensity score match among 142 patients. Importantly, these authors reported a trend toward improved survival for the adenocarcinoma group treated with paclitaxel, and they recommended a focus on this trend for future studies with large sample sizes. We confirmed these findings and report poorer disease-free survival in patients with adenocarcinoma/adenosquamous carcinoma than that in patients with squamous cell carcinoma-based 


\section{Study name}

$\begin{array}{lccccc} & \begin{array}{c}\text { Hazard } \\ \text { ratio }\end{array} & \begin{array}{c}\text { Lower } \\ \text { limit }\end{array} & \begin{array}{c}\text { Upper } \\ \text { limit }\end{array} & \text { Z-Value } & \text { p-Value } \\ \text { Kang et al. (2020) } & 1.520 & 1.065 & 2169 & 2307 & 0.021 \\ \text { Hu et al. (2018) } & 2.000 & 1.422 & 2814 & 3.979 & 0.000 \\ \text { Noh et al. (2014) } & 1.270 & 0.963 & 1.674 & 1.695 & 0.090 \\ \text { Katanyoo et al. (2012) } & 1.040 & 0.826 & 1.309 & 0.334 & 0.738 \\ & 1.387 & 1.052 & 1.828 & 2318 & 0.020\end{array}$

Hazard ratio and $95 \% \mathrm{Cl}$

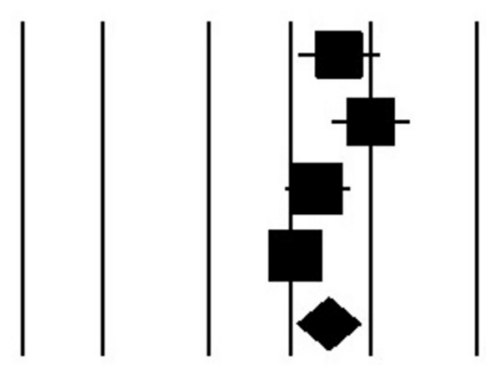

$\begin{array}{lllllll}0.1 & 0.2 & 0.5 & 1 & 2 & 5 & 10\end{array}$

FIGURE 8 | Forest plot for studies evaluating the overall survival in patients with differential tumor size.

\section{Study name}

Kang et al. (2020)

Hu et al. (2018)

Zhou et al. (2017)

Yoka et al. (2017)

Rose et al. (2014)

Chen et al. (2014)

Noh et al. (2014)

Katanyoo et al. (2012)

\section{Statistics for each study}

Hazard
ratio

2080

1.840

2.840

1.600

3.270

0.760

1.284

2.330

1.842

\section{Lower Upper} limit limit

1.236

3.499

1.434

2361

2.515

3.207

0.512

4.999

2.316

4.617

0.883

1.720

0.959

1.868

2906

2914
Z-Value

2.760

4.797

16.846

0.809

6.732

$-3.582$

1.676

7.504

2.612

p-Value
0.006
0.000
0.000
0.419
0.000
0.000
0.094
0.000
0.009

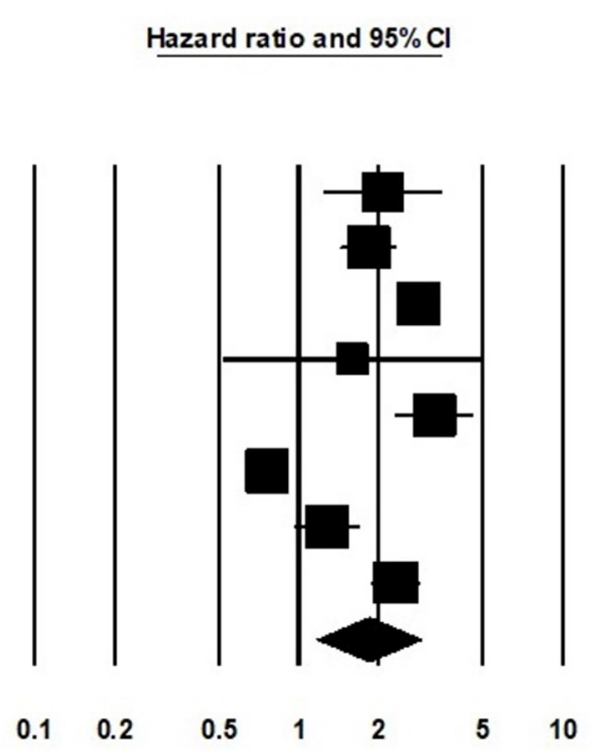

FIGURE 9 | Forest plot for studies evaluating the overall survival in patients with differential tumor stage.

cervical cancer $(\mathrm{HR}, 1.51)$ after definitive radiotherapy or concurrent chemoradiotherapy.

In terms of the overall survivals after the standard treatment for these cervical cancer types, we found a lack of consensus in the included studies. In a retrospective cohort study, Rose et al. (23) reported similar overall survivals among 1,671 patients with advanced-stage cervical cancer $(p=$ 0.45 ) regardless of the cancer subtype group and specially for the patients receiving cisplatin-based chemoradiation. Similarly, Katanyoo et al. (22) also reported non-significant differences (0.13) in the 5-year overall survivals between adenocarcinoma and squamous cell carcinoma groups receiving radiation therapy and concurrent chemotherapy. The authors mentioned that the similar overall survivals between the two histological subtypes remained even when they compared the tumor stages and sizes, and their respective treatments. On the other hand, Yokoi et al. (20) and $\mathrm{Hu}$ et al. (18) reported improved overall survivals for the squamous cell carcinoma group than for the adenocarcinoma/adenosquamous carcinoma group. They also mentioned that the patients with adenocarcinoma/adenosquamous cervical carcinoma had higher failure rates and relapse risks after definitive radiotherapy or concurrent chemoradiotherapy (18). Our results support those findings showing poorer overall survivals in patients with 
adenocarcinoma/adenosquamous cervical carcinoma than in those with squamous cell cervical carcinoma (HR, 1.41) after definitive radiotherapy or concurrent chemoradiotherapy.

Our study had some limitations. First, we were not able to pre-register this study in a systematic review repository such as PROSPERO York or Joanna Briggs Institute. We understand that this may raise concerns on the validity of our findings (37). However, we assure our readers that we made several attempts to register this review, but failed due to the extended registration times at the repositories owing to the COVID-19 pandemic. Second, all the included studies in our review were retrospective cohort studies, as a result we cannot exclude potential sources of biases. Third, our study did not evaluate the potential role of surgery in the evaluated outcomes. Fourth, the included studies reported survivals after differing follow-up periods after the definitive radiotherapy or concurrent chemoradiotherapy: Five studies reported the comparative survival outcomes after 5 years $(17,20-22,32)$, two after 3 years $(18,24)$, and one after 10 years (23). Therefore, we cannot rule out bias in our findings. Future studies are needed to address these limitations and validate our results.

In conclusion, we found survival differences among patients with cervical cancer after definitive radiotherapy or concurrent

\section{REFERENCES}

1. Shrestha AD, Neupane D, Vedsted P, Kallestrup P. Cervical cancer prevalence, incidence and mortality in low and middle income countries: a systematic review. Asian Pac J Cancer Prev. (2018) 19:319-24. doi: 10.22034/APJCP.2018.19.2.319

2. World Health Organization. Cervical Cancer. Available online at: https:// www.who.int/westernpacific/health-topics/cervical-cancer (accessed May 17, 2021).

3. Eifel PJ, Klopp AH, Berek JS, Konstantinopoulos PA. Cancer of the cervix, vagina, and vulva. In: DeVita VT, Rosenberg SA, Lawrence TS, editors. DeVita, Hellman, and Rosenberg's Cancer: Principles and Practice of Oncology, $10^{\text {th }} \mathrm{ed}$. London: Wolters Kluwer Health Adis (ESP) (2018). p. 1172-210.

4. The American Cancer Society. What Is Cervical Cancer? | Types of Cervical Cancer. Available online at: https://www.cancer.org/cancer/cervical-cancer/ about/what-is-cervical-cancer.html (accessed May 17, 2021).

5. Lemp JM, De Neve J-W, Bussmann H, Chen S, Manne-Goehler J, Theilmann $\mathrm{M}$, et al. Lifetime prevalence of cervical cancer screening in 55 low- and middle-income countries. JAMA. (2020) 324:153242. doi: 10.1001/jama.2020.16244

6. Nilima null, Puranik A, Shreenidhi SM, Rai SN. Spatial evaluation of prevalence, pattern and predictors of cervical cancer screening in India. Public Health. (2020) 178:124-36. doi: 10.1016/j.puhe.2019.09.008

7. Islami F, Fedewa SA, Jemal A. Trends in cervical cancer incidence rates by age, race/ethnicity, histological subtype, and stage at diagnosis in the United States. Prev Med. (2019) 123:316-23. doi: 10.1016/j.ypmed.2019.04.010

8. Bedell SL, Goldstein LS, Goldstein AR, Goldstein AT. Cervical cancer screening: past, present, and future. Sex Med Rev. (2020) 8:28-37. doi: 10.1016/j.sxmr.2019.09.005

9. Cuzick J, Mayrand MH, Ronco G, Snijders P, Wardle J. Chapter 10: new dimensions in cervical cancer screening. Vaccine. (2006) 24(Suppl 3):907. doi: 10.1016/j.vaccine.2006.05.122

10. Stumbar SE, Stevens M, Feld Z. Cervical cancer and its precursors: a preventative approach to screening, diagnosis, and management. Prim Care. (2019) 46:117-34. doi: 10.1016/j.pop.2018.10.011

11. Castanon A, Landy $R$, Sasieni PD. Is cervical screening preventing adenocarcinoma and adenosquamous carcinoma of chemoradiotherapy based on the cancer's histological subtype, tumor size, and tumor stage. These findings should be useful for clinicians because they synthesize the available evidence on the prognoses of the different cervical cancer subtypes after the standard management.

\section{DATA AVAILABILITY STATEMENT}

The original contributions presented in the study are included in the article/supplementary material, further inquiries can be directed to the corresponding author/s.

\section{AUTHOR CONTRIBUTIONS}

GY and JQ: conceptualized and designed the study. GY and FZ: did literature search and data collection. JQ and XW: analyzed the data. GY: wrote the paper. FZ and XW: reviewed and edited the manuscript. All authors read and approved the final manuscript.

\section{FUNDING}

The current study was funded by Huzhou Science and Technology Bureau (Grant Number: 2018GY01). the cervix? Int I Cancer. (2016) 139:1040-5. doi: 10.1002/ijc. 30152

12. Gien LT, Beauchemin M-C, Thomas G. Adenocarcinoma: a unique cervical cancer. Gynecol Oncol. (2010) 116:140-6. doi: 10.1016/j.ygyno.2009.09.040

13. van der Horst J, Siebers AG, Bulten J, Massuger LF, de Kok IM. Increasing incidence of invasive and in situ cervical adenocarcinoma in the Netherlands during 2004-2013. Cancer Med. (2017) 6:416-23. doi: 10.1002/cam4.971

14. Chen X, Zou H, Li H, Lin R, Su M, Zhang W, et al. Weekly versus triweekly cisplatin-based chemotherapy concurrent with radiotherapy in the treatment of cervical cancer: a meta-analysis. Int J Gynecol Cancer Off J Int Gynecol Cancer Soc. (2017) 27:344-9. doi: 10.1097/IGC.0000000000000883

15. Gupta S, Maheshwari A, Parab P, Mahantshetty U, Hawaldar R, Sastri Chopra S, et al. Neoadjuvant chemotherapy followed by radical surgery versus concomitant chemotherapy and radiotherapy in patients with stage IB2, IIA, or IIB squamous cervical cancer: a randomized controlled trial. J Clin Oncol Off J Am Soc Clin Oncol. (2018) 36:1548-55. doi: 10.1200/JCO.2017.75.9985

16. Petrelli F, De Stefani A, Raspagliesi F, Lorusso D, Barni S. Radiotherapy with concurrent cisplatin-based doublet or weekly cisplatin for cervical cancer: a systematic review and meta-analysis. Gynecol Oncol. (2014) 134:16671. doi: 10.1016/j.ygyno.2014.04.049

17. Chen JL-Y, Huang C-Y, Huang Y-S, Chen R-J, Wang C-W, Chen Y-H, et al. Differential clinical characteristics, treatment response and prognosis of locally advanced adenocarcinoma/adenosquamous carcinoma and squamous cell carcinoma of cervix treated with definitive radiotherapy. Acta Obstet Gynecol Scand. (2014) 93:661-8. doi: 10.1111/aogs.12383

18. Hu K, Wang W, Liu X, Meng Q, Zhang F. Comparison of treatment outcomes between squamous cell carcinoma and adenocarcinoma of cervix after definitive radiotherapy or concurrent chemoradiotherapy. Radiat Oncol Lond Engl. (2018) 13:249. doi: 10.1186/s13014-018-1197-5

19. Lee J-Y, Kim YT, Kim S, Lee B, Lim MC, Kim J-W, et al. Prognosis of cervical cancer in the era of concurrent chemoradiation from national database in Korea: a comparison between squamous cell carcinoma and adenocarcinoma. PLoS ONE. (2015) 10:e0144887. doi: 10.1371/journal.pone.01 44887

20. Yokoi E, Mabuchi S, Takahashi R, Matsumoto Y, Kuroda H, Kozasa K, et al. Impact of histological subtype on survival in patients with locally advanced cervical cancer that were treated with definitive radiotherapy: 
adenocarcinoma/adenosquamous carcinoma versus squamous cell carcinoma. J Gynecol Oncol. (2017) 28:e19. doi: 10.3802/jgo.2017.28.e19

21. Kang J-H, Cho WK, Yeo HJ, Jeong SY, Noh JJ, Shim JI, et al. Prognostic significance of tumor regression rate during concurrent chemoradiotherapy in locally advanced cervix cancer: analysis by radiation phase and histologic type. J Clin Med. (2020) 9:3471. doi: 10.3390/jcm9113471

22. Katanyoo K, Sanguanrungsirikul S, Manusirivithaya S. Comparison of treatment outcomes between squamous cell carcinoma and adenocarcinoma in locally advanced cervical cancer. Gynecol Oncol. (2012) 125:2926. doi: 10.1016/j.ygyno.2012.01.034

23. Rose PG, Java JJ, Whitney CW, Stehman FB, Lanciano R, Thomas GM. Locally advanced adenocarcinoma and adenosquamous carcinomas of the cervix compared to squamous cell carcinomas of the cervix in gynecologic oncology group trials of cisplatin-based chemoradiation. Gynecol Oncol. (2014) 135:208-12. doi: 10.1016/j.ygyno.2014.08.018

24. Zhou J, Wu S-G, Sun J-Y, Li F-Y, Lin H-X, Chen Q-H, et al. Comparison of clinical outcomes of squamous cell carcinoma, adenocarcinoma, and adenosquamous carcinoma of the uterine cervix after definitive radiotherapy: a population-based analysis. J Cancer Res Clin Oncol. (2017) 143:11522. doi: 10.1007/s00432-016-2246-9

25. Moher D, Liberati A, Tetzlaff J, Altman DG, PRISMA Group. Preferred reporting items for systematic reviews and meta-analyses: the PRISMA statement. PLoS Med. (2009) 6:e1000097. doi: 10.1371/journal.pmed.1000097

26. Stang A. Critical evaluation of the Newcastle-Ottawa scale for the assessment of the quality of nonrandomized studies in meta-analyses. Eur J Epidemiol. (2010) 25:603-5. doi: 10.1007/s10654-010-9491-Z

27. Bax L, Yu L-M, Ikeda N, Moons KGM. A systematic comparison of software dedicated to meta-analysis of causal studies. BMC Med Res Methodol. (2007) 7:40. doi: 10.1186/1471-2288-7-40

28. Higgins JPT, Thompson SG, Spiegelhalter DJ. A re-evaluation of random-effects meta-analysis. J R Stat Soc A Stat Soc. (2009) 172:137-59. doi: 10.1111/j.1467-985X.2008.00552.x

29. Higgins JPT, Thompson SG. Quantifying heterogeneity in a meta-analysis. Stat Med. (2002) 21:1539-58. doi: 10.1002/sim.1186

30. Hozo SP, Djulbegovic B, Hozo I. Estimating the mean and variance from the median, range, and the size of a sample. BMC Med Res Methodol. (2005) 5:13. doi: 10.1186/1471-2288-5-13

31. Duval S, Tweedie R. Trim and fill: a simple funnel-plot-based method of testing and adjusting for publication bias in meta-analysis. Biometrics. (2000) 56:455-63. doi: 10.1111/j.0006-341x.2000.00455.x

32. Noh JM, Park W, Kim YS, Kim J-Y, Kim HJ, Kim J, et al. Comparison of clinical outcomes of adenocarcinoma and adenosquamous carcinoma in uterine cervical cancer patients receiving surgical resection followed by radiotherapy: a multicenter retrospective study (KROG 13-10). Gynecol Oncol. (2014) 132:618-23. doi: 10.1016/j.ygyno.2014.01.043

33. LaVigne AW, Triedman SA, Randall TC, Trimble EL, Viswanathan AN. Cervical cancer in low and middle income countries: addressing barriers to radiotherapy delivery. Gynecol Oncol Rep. (2017) 22:1620. doi: 10.1016/j.gore.2017.08.004

34. Mwaka AD, Wabinga HR, Mayanja-Kizza H. Mind the gaps: a qualitative study of perceptions of healthcare professionals on challenges and proposed remedies for cervical cancer help-seeking in post conflict northern Uganda. BMC Fam Pract. (2013) 14:193. doi: 10.1186/1471-2296-14-193

35. van Meir H, Kenter GG, Burggraaf J, Kroep JR, Welters MJP, Melief CJM, et al. The need for improvement of the treatment of advanced and metastatic cervical cancer, the rationale for combined chemo-immunotherapy. Anticancer Agents Med Chem. (2014) 14:190-203. doi: 10.2174/18715206113136660372

36. Huang Y-T, Wang C-C, Tsai C-S, Lai C-H, Chang T-C, Chou H$\mathrm{H}$, et al. Clinical behaviors and outcomes for adenocarcinoma or adenosquamous carcinoma of cervix treated by radical hysterectomy and adjuvant radiotherapy or chemoradiotherapy. Int J Radiat Oncol Biol Phys. (2012) 84:420-7. doi: 10.1016/j.ijrobp.2011.12.013

37. PLoS Medicine Editors. Best practice in systematic reviews: the importance of protocols and registration. PLoS Med. (2011) 8:e1001009. doi: 10.1371/journal.pmed.1001009

Conflict of Interest: The authors declare that the research was conducted in the absence of any commercial or financial relationships that could be construed as a potential conflict of interest.

Publisher's Note: All claims expressed in this article are solely those of the authors and do not necessarily represent those of their affiliated organizations, or those of the publisher, the editors and the reviewers. Any product that may be evaluated in this article, or claim that may be made by its manufacturer, is not guaranteed or endorsed by the publisher.

Copyright (C) 2022 Yao, Qiu, Zhu and Wang. This is an open-access article distributed under the terms of the Creative Commons Attribution License (CC BY). The use, distribution or reproduction in other forums is permitted, provided the original author(s) and the copyright owner(s) are credited and that the original publication in this journal is cited, in accordance with accepted academic practice. No use, distribution or reproduction is permitted which does not comply with these terms. 